Pacific Journal of Mathematic 


\title{
NORMS AND INEQUALITIES FOR CONDITION NUMBERS
}

\author{
Albert W. Marshall and Ingram Olkin
}

The condition number $c_{\varphi}$ of a nonsingular matrix $A$ is defined by $c_{\varphi}(A)=\varphi(A) \varphi\left(A^{-1}\right)$ where ordinarily $\varphi$ is a norm. It was proved by 0 . Taussky-Todd that $(c) c_{\varphi}(A) \leqq c_{\varphi}\left(A A^{*}\right)$ when $\varphi(A)=\left(\operatorname{tr} A A^{*}\right)^{1 / 2}$ and when $\varphi(A)$ is the maximum absolute characteristic root of $A$. It is shown that $(c)$ holds whenever $\varphi$ is a unitarily invariant norm, i.e., whenever $\varphi$ satisfies $\varphi(A)>0$ for $A \neq 0 ; \varphi(\alpha A)=|\alpha| \varphi(A)$ for complex $\alpha$; $\varphi(A+B) \leqq \varphi(A)+\varphi(B) ; \varphi(A)=\varphi(A U)=\varphi(A U)$ for all unitary $U$. If in addition, $\varphi\left(E_{i j}\right)=1$, where $E_{i j}$ is the matrix with one in the $(i, j)$ th place and zeros elsewhere, then $c_{\varphi}(A) \geqq\left[c_{\varphi}\left(A A^{*}\right)\right]^{1 / 2}$. Generalizations are obtained by exploiting the relation between unitarily invariant norms and symmetric gauge functions. However, it is shown that (c) is independent of the usual norm axioms.

1. Introduction. The genesis of this study is the proposition that under certain conditions, the matrix $A A^{*}$ is more "ill-conditioned" than $A$. More precisely, the condition number $c_{\varphi}(A)$ is defined for nonsingular matrices $A$ as

$$
c_{\varphi}(A)=\varphi(A) \varphi\left(A^{-1}\right)
$$

where ordinarily $\varphi$ is a norm. The statement concerning ill-conditioning of $A A^{*}$ is the inequality

$$
c_{\varphi}(A) \leqq c_{\varphi}\left(A A^{*}\right)
$$

Where $\varphi(A)$ is the maximum absolute characteristic root of $A$ and where $\varphi(A)=\left(\operatorname{tr} A A^{*}\right)^{1 / 2}$, inequality $(c)$ was proved by 0. Taussky-Todd [7]. This raises the question of whether $(c)$ is true for all norms. In this paper, we show that quite the contrary is true; $(c)$ is independent of the usual norm axioms. However, we also prove that $(c)$ does hold for a quite general class of norms.

In the course of proving these results, we obtain some inequalities for symmetric gauge functions, which may be of independent interest.

2. Gauge functions and matrix norms. We call $\varphi$ a matrix norm if

$$
\varphi(A)>0 \quad \text { when } A \neq 0 \text {, }
$$

Received March 10, 1964. 


$$
\begin{gathered}
\varphi(\alpha A)=|\alpha| \varphi(A) \quad \text { for complex } \alpha, \\
\varphi(A+B) \leqq \varphi(A)+\varphi(B) .
\end{gathered}
$$

In addition to these basic axioms, various other conditions are sometimes imposed:

$$
\varphi\left(E_{i j}\right)=1,
$$

where $E_{i j}$ is the matrix with one in the $(i, j)$ th position and zero elsewhere,

$$
\varphi(A B) \leqq \varphi(A) \varphi(B),
$$

$$
\varphi(A)=\varphi(U A)=\varphi(A U)
$$

for all unitary matrices $U$.

If $\varphi$ satisfies aI, aII, aIII, and aVI, $\varphi$ is called a unitarily invariant norm.

There is an important connection between unitarily invariant norms and symmetric gauge functions. A function $\Phi$ on a complex vector space is called a gauge function if

$$
\Phi(u)>0 \quad \text { when } u \neq 0,
$$

$$
\begin{gathered}
\Phi(\alpha u)=|\alpha| \Phi(u) \quad \text { for complex } \alpha, \\
\Phi(u+v) \leqq \Phi(u)+\Phi(v) .
\end{gathered}
$$

Often it is convenient to assume, in addition, that

$$
\Phi\left(e_{i}\right)=1,
$$

where $e_{i}$ is the vector with one in the $i$ th place and zero elsewhere. If, in addition to bI, bII, and bIII,

$$
\Phi\left(u_{1}, \cdots, u_{n}\right)=\Phi\left(\varepsilon_{1} u_{i_{1}}, \cdots, \varepsilon_{n} u_{i_{n}}\right)
$$

whenever $\varepsilon_{j}= \pm 1$ and $\left(i_{1}, \cdots, i_{n}\right)$ is a permutation of $(1, \cdots, n)$, then $\Phi$ is called a symmetric gauge function.

It was noted by Von Neumann [8] that a norm $\varphi$ is unitarily invariant if and only if there exists a symmetric gauge function $\Phi$ such that $\varphi(A)=\Phi(\alpha)$ for all $A$, where $\alpha_{1}^{2}, \cdots, \alpha_{n}^{2}$ are the eigenvalues of $A A^{*}$.

If $\Phi$ is a symmetric gauge function and $u, v$ satisfy $u_{i} \leqq v_{i}, i=$ $1, \cdots, n$, then it follows $[6, \mathrm{p} .85]$ that

$$
\Phi\left(u_{1}, \cdots, u_{n}\right) \leqq \Phi\left(v_{1}, \cdots, v_{n}\right) \text {. }
$$

If $\Phi$ is a symmetric gauge function satisfying bIV, then $[6, p .86]$

$$
\max _{i}\left|u_{i}\right| \leqq \Phi\left(u_{1}, \cdots, u_{n}\right) \leqq \sum_{i=1}^{n}\left|u_{i}\right|
$$


If $\varphi$ is the unitarily invariant matrix norm determined by $\Phi$ as above, then it follows that

$$
\begin{aligned}
\frac{\varphi(A B)}{\varphi(A) \varphi(B)} & \leqq \frac{\sum_{i=1}^{n} \lambda_{i}\left(A B B^{*} A^{*}\right)}{\left[\max _{i} \lambda_{i}\left(A A^{*}\right)\right]\left[\max _{j} \lambda_{j}\left(B B^{*}\right)\right]} \\
& \leqq \frac{n \max _{i} \lambda_{i}\left(B B^{*} A^{*} A\right)}{\left[\max _{i} \lambda_{i}\left(A A^{*}\right)\right]\left[\max _{j} \lambda_{j}\left(B B^{*}\right)\right]} \leqq n,
\end{aligned}
$$

where $\lambda_{i}(M)$ are the eigenvalues of $M$. Thus, for any $k \geqq n, k \varphi$ is a unitarily invariant matrix norm also satisfying $\mathrm{aV}$. Of course, $\varphi$ itself satisfies aIV (since $\Phi$ satisfies bIV), and this property is destroyed by the renormalization.

3. The condition number inequality.

THEOREM 3.1. If $\varphi$ is a unitarily invariant norm, then

$$
c_{\varphi}(A) \leqq c_{\varphi}\left(A A^{*}\right) .
$$

If $\Phi$ is a symmetric gauge function which determines $\varphi$, then we may rewrite (c) in the form

$$
\Phi\left(\alpha_{1}, \cdots, \alpha_{n}\right) \Phi\left(\alpha_{1}^{-1}, \cdots, \alpha_{n}^{-1}\right) \leqq \Phi\left(\alpha_{1}^{2}, \cdots, \alpha_{n}^{2}\right) \Phi\left(\alpha_{1}^{-2}, \cdots, \alpha_{n}^{-2}\right) .
$$

Thus, Theorem 3.1 is a very special case of

THEOREM 3.2. If $\Phi$ is a symmetric gauge function, then $\Phi\left(\alpha_{1}^{r}, \cdots, \alpha_{n}^{r}\right) \Phi\left(\alpha_{1}^{-r}, \cdots, \alpha_{n}^{-r}\right)$ is increasing in $r>0$, where $\alpha_{i}>0$.

The proof of Theorem 3.2 is embodied in the lemmas below.

Following [2] we say $\left(a_{1}, \cdots, a_{n}\right)$ is majorized by $\left(b_{1}, \cdots, b_{n}\right)$, written (a) $\prec$ (b), if

(i ) $a_{1} \geqq \cdots \geqq a_{n}>0, b_{1} \geqq \cdots \geqq b_{n}>0$,

(ii) $\sum_{1}^{k} a_{i} \leqq \sum_{1}^{k} b_{i}, \quad k=1, \cdots, n-1$,

(iii) $\sum_{1}^{n} a_{i}=\sum_{1}^{n} b_{i}$.

LEMMA 3.3. If $(\mathrm{a}) \prec(\mathrm{b})$, and $\Phi$ is a symmetric gauge function, then

$$
\begin{gathered}
\Phi\left(a_{1}, \cdots, a_{n}\right) \leqq \Phi\left(b_{1}, \cdots, b_{n}\right), \\
\Phi\left(a_{1}^{-1}, \cdots, a_{n}^{-1}\right) \leqq \Phi\left(b_{1}^{-1}, \cdots, b_{n}^{-1}\right) .
\end{gathered}
$$

Proof. Proofs of (3.1) have been given by Fan [1] and Ostrowski 
[3]; by an argument similar to that of Fan, we prove (3.2).

First, note that we can assume for $h$ and $j$ fixed, $h<j$,

$$
a_{h}=\alpha b_{h}+(1-\alpha) b_{j}, a_{j}=(1-\alpha) b_{h}+\alpha b_{j}, a_{i}=b_{i}, i \neq h, j .
$$

That this is true follows from the fact that if (a) $\prec(b)$, then $a$ car be derived from $b$ by successive applications of a finite number of transformations of the form (3.3) (see [2, p. 47]).

Let $\widetilde{b}=\left(b_{1}, \cdots, b_{h-1}, b_{j}, b_{h+1}, \cdots, b_{j-1}, b_{h}, b_{j+1}, \cdots, b_{n}\right)$, so that $\Phi\left(b_{1}, \cdots, b_{n}\right)$ $=\Phi\left(\widetilde{b}_{1}, \cdots, \widetilde{b}_{n}\right)$. By convexity,

$$
\left(\alpha b_{i}+(1-\alpha) \tilde{b}_{i}\right)^{-1} \leqq \alpha b_{i}^{-1}+(1-\alpha) \tilde{b}_{i}^{-1} .
$$

Then using (2.1) and the convexity of $\Phi$, it follows that

$$
\begin{aligned}
\Phi\left(a_{1}^{-1}, \cdots, a_{n}^{-1}\right) & =\Phi\left[\left(\alpha b_{1}+(1-\alpha) \widetilde{b}_{1}\right)^{-1}, \cdots,\left(\alpha b_{n}+(1-\alpha) \widetilde{b}_{n}\right)^{-1}\right] \\
& \leqq \Phi\left(\alpha b_{1}^{-1}+(1-\alpha) \widetilde{b}_{1}^{-1}, \cdots, \alpha b_{n}^{-1}+(1-\alpha) \tilde{b}_{n}^{-1}\right) \\
& \leqq \alpha \Phi\left(b_{1}^{-1}, \cdots, b_{n}^{-1}\right)+(1-\alpha) \Phi\left(\widetilde{b}_{1}^{-1}, \cdots, \widetilde{b}_{n}^{-1}\right) . \|
\end{aligned}
$$

As a consequence of Lemma 3.3., we have that if (a) $\prec$ (b) them

$$
\Phi\left(a_{1}, \cdots, a_{n}\right) \Phi\left(a_{1}^{-1}, \cdots, a_{n}^{-1}\right) \leqq \Phi\left(b_{1}, \cdots, b_{n}\right) \Phi\left(b_{1}^{-1}, \cdots, b_{n}^{-1}\right) \text {. }
$$

The proof of Theorem 3.2 is completed by the following

LEMMA 3.4. If $\alpha_{1} \geqq \cdots \geqq \alpha_{n}>0$ and $a_{i}=\alpha_{i}^{r} / \Sigma \alpha_{j}^{r}, b_{i}=\alpha_{i}^{s} / \Sigma \alpha_{j,}^{s}$ $0<r<s$, then (a) $\prec(\mathrm{b})$.

Proof. We must show that for all $k$,

$$
\frac{\sum_{1}^{k} \alpha_{i}^{r}}{\sum_{1}^{n} \alpha_{i}^{r}} \leqq \frac{\sum_{1}^{k} \alpha_{i}^{s}}{\sum_{1}^{n} \alpha_{i}^{s}}, \quad r<s,
$$

which is true if and only if

$$
\sum_{1}^{k} \alpha_{i}^{s} \sum_{k+1}^{n} \alpha_{j}^{r}-\sum_{1}^{k} \alpha_{i}^{r} \sum_{k+1}^{n} \alpha_{j}^{s}=\sum_{i=1}^{k} \alpha_{i}^{r} \sum_{j=k+1}^{n} \alpha_{j}^{r}\left(\alpha_{i}^{s-r}-\alpha_{j}^{s-r}\right) \geqq 0 .
$$

The latter follows from $\alpha_{i} \geqq \alpha_{j}, i<j$. |

Observe that by (3.1) and Lemma 3.4, we have

$$
\frac{\Phi\left(\alpha_{1}^{r}, \cdots, \alpha_{n}^{r}\right)}{\Phi\left(\alpha_{1}^{s}, \cdots, \alpha_{n}^{s}\right)} \leqq \frac{\Sigma \alpha_{i}^{r}}{\Sigma \alpha_{i}^{s}} .
$$

In view of (2.2), it is perhaps natural to expect that

$$
\frac{\alpha_{1}^{r}}{\alpha_{1}^{s}} \leqq \frac{\Phi\left(\alpha_{1}^{r}, \cdots, \alpha_{n}^{r}\right)}{\Phi\left(\alpha_{1}^{s}, \cdots, \alpha_{n}^{s}\right)} \leqq \frac{\Sigma \alpha_{i}^{r}}{\Sigma \alpha_{i}^{s}}, 0<r<s, \alpha_{1} \geqq \cdots \geqq \alpha_{n}>0,
$$


for any symmetric gauge function $\Phi$. To see this we need only prove the left hand inequality, which may be written in the form

$$
\Phi\left(\left[\frac{\alpha_{1}}{\alpha_{1}}\right]^{s}, \cdots,\left[\frac{\alpha_{n}}{\alpha_{1}}\right]^{s}\right) \leqq \Phi\left(\left[\frac{\alpha_{1}}{\alpha_{1}}\right]^{r}, \cdots,\left[\frac{\alpha_{n}}{\alpha_{1}}\right]^{r}\right),
$$

and which is a consequence of (2.1).

An interesting counterpart to Theorem 3.2 can be obtained from (3.4).

THEOREM 3.5. If $\Phi$ is a symmetric gauge function satisfying bIV, then $\left[\Phi\left(\alpha_{1}^{r}, \cdots, \alpha_{n}^{r}\right)\right]^{1 / r}$ is decreasing in $r>0$ whenever $\alpha_{i}>0$, $i=1,2, \cdots, n$. Thus $\left[\Phi\left(\alpha_{1}^{r}, \cdots, \alpha_{n}^{r}\right) \Phi\left(\alpha_{1}^{-r}, \cdots, \alpha_{n}^{-r}\right)\right]^{1 / r}$ is decreasing in $r>0$.

Proof. We have that

$$
1 \leqq \Phi\left(\left[\frac{\alpha_{1}}{\alpha_{1}}\right]^{s}, \cdots,\left[\frac{\alpha_{n}}{\alpha_{1}}\right]^{s}\right) \leqq \Phi\left(\left[\frac{\alpha_{1}}{\alpha_{1}}\right]^{r}, \cdots,\left[\frac{\alpha_{n}}{\alpha_{1}}\right]^{r}\right),
$$

the first inequality by bIV and (2.1). The second inequality is (3.5). Thus

$$
\begin{aligned}
\left\{\Phi\left(\left[\frac{\alpha_{1}}{\alpha_{1}}\right]^{s}, \cdots,\left[\frac{\alpha_{n}}{\alpha_{1}}\right]^{s}\right)\right\}^{r} & \leqq\left\{\Phi\left(\left[\frac{\alpha_{1}}{\alpha_{1}}\right]^{r}, \cdots,\left[\frac{\alpha_{n}}{\alpha_{1}}\right]^{r}\right)\right\}^{r} \\
& \leqq\left\{\Phi\left(\left[\frac{\alpha_{1}}{\alpha_{1}}\right]^{r}, \cdots,\left[\frac{\alpha_{n}}{\alpha_{1}}\right]^{r}\right)\right\}^{s}
\end{aligned}
$$

so that

$$
\left\{\Phi\left(\left[\frac{\alpha_{1}}{\alpha_{1}}\right]^{s}, \cdots,\left[\frac{\alpha_{n}}{\alpha_{1}}\right]^{s}\right)\right\}^{1 / s} \leqq\left\{\Phi\left(\left[\frac{\alpha_{1}}{\alpha_{1}}\right]^{r}, \cdots,\left[\frac{\alpha_{n}}{\alpha_{1}}\right]^{r}\right)\right\}^{1 / r} .
$$

The theorem now follows from bII.

Theorem 3.5 can, of course, be specialized to yield a kind of converse to (c).

THEOREM 3.6. If $\varphi$ is a unitarily invariant norm satisfying aIV, then

$$
\left[c_{\varphi}\left(A A^{*}\right)\right]^{1 / 2} \leqq c_{\varphi}(A) .
$$

Condition $\left(\mathrm{c}^{*}\right)$ can also be obtained under somewhat different hypotheses. In particular, if $\varphi$ satisfies $a V$, then

$$
\begin{aligned}
c_{\varphi}\left(A A^{*}\right) & =\varphi\left(A A^{*}\right) \varphi\left(\left(A A^{*}\right)^{-1}\right) \\
& \leqq \varphi(A) \varphi\left(A^{-1}\right) \varphi\left(A^{*}\right) \varphi\left(A^{*-1}\right)=c_{\varphi}(A) c_{\varphi}\left(A^{*}\right) .
\end{aligned}
$$


If also $\varphi(A)=\varphi\left(A^{*}\right)$, then ( $\left.\mathrm{c}^{*}\right)$ follows. Of course, $\varphi(A)=\varphi\left(A^{*}\right)$ if $\varphi$ is unitarily invariant.

4. Independence of the norm axioms and (c). It is our purpose here to show that the condition number inequality (c) does not follow from the usual norm axioms aI - aV. In fact, aII, aIII, aIV, $\mathrm{aV}$ and (c) are independent.

REMARK. It has been shown by Ostrowski [4] that aI is implied by aII, aIII, aV, together with $\varphi(A) \not \equiv 0$, so that aI is not included in the list of independent properties. Rella [5] has shown that aII, aIII, aIV and aV are independent, and we add (c) to this list.

The results which prove the independence of aII $-\mathrm{aV}$ and (c) are summarized in the following table, where $+(-)$ indicates that a property is true (false).

\begin{tabular}{c||c|c|c|c|c|}
$\varphi(A)$ & aII & aIII & aIV & aV & (c) \\
\hline 1 & - & + & + & + & + \\
\hline $\begin{array}{c}(\operatorname{rank} A)\left(\operatorname{tr} A A^{*}\right)^{1 / 2} \\
n \max \left|a_{i j}\right|\end{array}$ & - & + & + & + \\
\hline $\max \left|a_{i j}\right|$ & + & + & - & + & + \\
$\Sigma\left|a_{i j}\right|$ & + & + & + & - & + \\
\hline+ & + & + & + & - \\
\hline
\end{tabular}

An example which serves in the last line of the table just as well as $\Sigma\left|a_{i j}\right|$ is the norm $\max _{i} \sum_{j}\left|a_{i j}\right|=\sup _{x} \Phi(x A) / \Phi(x)$, where $\Phi(x)=$ $\sum_{i}\left|x_{i}\right|$. Norms of this form are called "subordinate" or "lub" norms, and in this case $\Phi$ is a symmetric guage function.

The remainder of this paper is devoted to proving the propositions indicated in the table.

The results for $\varphi(A) \equiv 1$ are obvious, so we begin by considering $\varphi(A)=(\operatorname{rank} A)\left(\operatorname{tr} A A^{*}\right)^{1 / 2}$. In this case, aII and aIV are obvious, and (c) follows from Theorem 3.1, since $\left(\operatorname{tr} A A^{*}\right)^{1 / 2}$ is unitarily invariant. As is well known, $\left(\operatorname{tr} A A^{*}\right)^{1 / 2}$ satisfies aV; this together with rank $A B \leqq$ $(\operatorname{rank} A)(\operatorname{rank} B)$ yields aV for $\varphi(A)=(\operatorname{rank} A)\left(\operatorname{tr} A A^{*}\right)^{1 / 2}$. That aIII is violated may be seen by taking $A=I$ and $B$ the matrix with a unit in the $(1,1)$ th place and zeros elsewhere.

For $\varphi(A)=n \max _{i, j}\left|a_{i j}\right|$ and $\max _{i, j}\left|a_{i j}\right|$ the first four columns of the table are well known, and we need only prove (c). Let $e_{i}$ be the row vector with one in the $i$ th position and zero elsewhere. Denote $M^{-1}=\left(m^{i j}\right)$ where $M=\left(m_{i j}\right)$, and let $U=A A^{*}$. By Cauchy's inequality, 


$$
\begin{aligned}
\left|a_{i j}\right|\left|a^{\alpha \beta}\right| & =\left|e_{i} A e_{j}^{*}\right|\left|e_{\alpha} A^{-1} e_{\beta}^{*}\right| \leqq\left[\left(e_{i} U e_{i}^{*}\right)\left(e_{j} e_{j}^{*}\right)\left(e_{\alpha} e_{\alpha}^{*}\right)\left(e_{\beta} U^{-1} e_{\beta}^{*}\right)\right]^{1 / 2} \\
& =\left(u_{i i} u^{\beta \beta}\right)^{1 / 2}
\end{aligned}
$$

Hence,

$$
\max _{i j}\left|a_{i j}\right| \max _{\alpha \beta}\left|\alpha^{\alpha \beta}\right| \leqq\left(\max _{i}\left|u_{i i}\right| \max _{\alpha}\left|u^{\alpha \alpha}\right|\right)^{1 / 2},
$$

or

$$
c_{\varphi}(A) \leqq\left[c_{\varphi}\left(A A^{*}\right)\right]^{1 / 2} .
$$

Since $U=A A^{*}$ is positive semi-definite,

$$
u_{i i} u^{i i}=\left(e_{i} U e_{i}^{*}\right)\left(e_{i} U^{-1} e_{i}^{*}\right) \geqq\left(e_{i} e_{i}^{*}\right)^{2}=1,
$$

and it follows that $c_{\varphi}\left(A A^{*}\right) \geqq 1$. Thus, we have that

$$
c_{\varphi}(A) \leqq\left[c_{\varphi}\left(A A^{*}\right)\right]^{1 / 2} \leqq c_{\varphi}\left(A A^{*}\right),
$$

which gives $(\mathrm{c})$.

Note that the left inequality of (4.1) is a reversal of inequality $\left(\mathrm{c}^{*}\right)$. That (4.1) also holds if $\varphi(A)$ is the maximum of the absolute values of the characteristic values of $A$ was proved by O. TausskyTodd [6].

Since the first four columns of the table are well known for $\varphi(A)=$ $\Sigma\left|a_{i j}\right|$, we again need consider only (c). If $A=\left(\begin{array}{cc}B & 0 \\ 0 & 2 I\end{array}\right)$, where $B=$ $\left(\begin{array}{cc}2 & 1 \\ -1 & 2\end{array}\right)$. Then (c) is violated. This same example shows that (c) is violated for $\varphi(A)=\max _{i} \sum_{j}\left|a_{i j}\right|$.

\section{BIBLIOGRAPHY}

1. K. Fan, Maximum properties and inequalities for the eigenvalues of completely continuous operators, Proc. Nat. Acad. Sci. U.S.A. 37, (1951) 760-766.

2. G. H. Hardy, J. E. Littlewood and G. Pólya, Inequalities (2nd. Ed.), Cambridge University Press, Cambridge. (1959).

3. A. Ostrowski, Sur quelques applications des fonctions convexes et concaves au sens de I. Schur, J. Math. Pure Appl. 31, (1952), 253-292.

4. — Über Normen von Matrizen, Math. Z., 63, (1955), 2-18.

5. T. Rella, Über den absoluten Betrig von Matrizen, Proc. of the International Congress of Mathematicians, Oslo, (1936). 29-31.

6. R. Schatten, A theory of cross-spaces, Princeton University Press, Princeton (1950).

7. O. Taussky-Todd, Note on the condition of matrices, Math. Tables Aids Comput., 4, (1950), 111-112.

8. Von Neumann, Some matrix-inequalities and metrization of matric-space, Tomsk. Univ., Rev. 1, (1937) 286-300, (in Collected Works, Vol. IV, Pergamon Press, 1962). 



\section{PACIFIC JOURNAL OF MATHEMATICS}

\section{EDITORS}

H. Samelson

Stanford University

Stanford, California

R. M. Blumenthal

University of Washington

Seattle, Washington 98105
J. Dugundu

University of Southern California Los Angeles, California 90007

*Richard Arens

University of California

Los Angeles, California 90024

\section{ASSOCIATE EDITORS}
E. F. BECKENBACH
B. H. NeumanN
F. WOLF
K. YOSIDA

\section{SUPPORTING INSTITUTIONS}

UNIVERSITY OF BRITISH COLUMBIA CALIFORNIA INSTITUTE OF TECHNOLOGY UNIVERSITY OF CALIFORNIA MONTANA STATE UNIVERSITY

UNIVERSITY OF NEVADA

NEW MEXICO STATE UNIVERSITY

OREGON STATE UNIVERSITY

UNIVERSITY OF OREGON

OSAKA UNIVERSITY

UNIVERSITY OF SOUTHERN CALIFORNIA
STANFORD UNIVERSITY

UNIVERSITY OF TOKYO

UNIVERSITY OF UTAH

WASHINGTON STATE UNIVERSITY

UNIVERSITY OF WASHINGTON

AMERICAN MATHEMATICAL SOCIETY CALIFORNIA RESEARCH CORPORATION SPACE TECHNOLOGY LABORATORIES NAVAL ORDNANCE TEST STATION 


\section{Pacific Journal of Mathematics}

\section{Vol. 15, No. $1 \quad$ September, 1965}

Donald Charles Benson, Unimodular solutions of infinite systems of linear

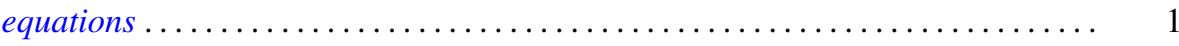

Richard Earl Block, Transitive groups of collineations on certain designs . . . . . . 13

Barry William Boehm, Existence of best rational Tchebycheff approximations .... . 19

Joseph Patrick Brannen, A note on Hausdorff's summation methods . . . . . . . . . . 29

Dennison Robert Brown, Topological semilattices on the two-cell ............ 35

Peter Southcott Bullen, Some inequalities for symmetric means . . . . . . . . . . 47

David Geoffrey Cantor, On arithmetic properties of coefficients of rational

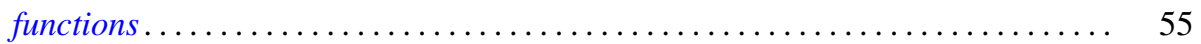

Luther Elic Claborn, Dedekind domains and rings of quotients . . . . . . . . . 59

Allan Clark, Homotopy commutativity and the Moore spectral sequence ........ 65

Allen Devinatz, The asymptotic nature of the solutions of certain linear systems of

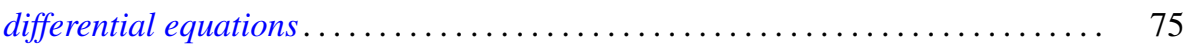

Robert E. Edwards, Approximation by convolutions ................... 85

Theodore William Gamelin, Decomposition theorems for Fredholm operators . . . . . 97

Edmond E. Granirer, On the invariant mean on topological semigroups and on

topological groups .................................. 107

Noel Justin Hicks, Closed vector fields . . . . . . . . . . . . . . . . . . . 141

Charles Ray Hobby and Ronald Pyke, Doubly stochastic operators obtained from

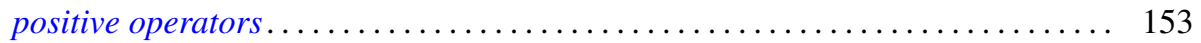

Robert Franklin Jolly, Concerning periodic subadditive functions . . . . . . . . . 159

Tosio Kato, Wave operators and unitary equivalence . . . . . . . . . . . . . . 171

Paul Katz and Ernst Gabor Straus, Infinite sums in algebraic structures . . . . . . . 181

Herbert Frederick Kreimer, Jr., On an extension of the Picard-Vessiot theory ...... 191

Radha Govinda Laha and Eugene Lukacs, On a linear form whose distribution is

identical with that of a monomial ......................... 207

Donald A. Ludwig, Singularities of superpositions of distributions . . . . . . . . . 215

Albert W. Marshall and Ingram Olkin, Norms and inequalities for condition

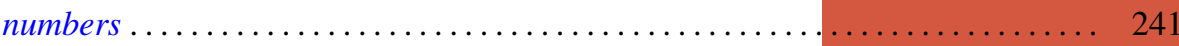

Horace Yomishi Mochizuki, Finitistic global dimension for rings . . . . . . . . . . 249

Robert Harvey Oehmke and Reuben Sandler, The collineation groups of division

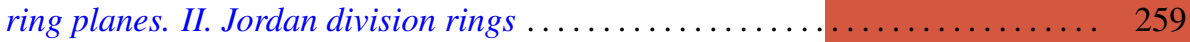

George H. Orland, On non-convex polyhedral surfaces in $E^{3} \ldots \ldots \ldots \ldots \ldots \ldots \ldots 267$

Theodore G. Ostrom, Collineation groups of semi-translation planes . . . . . . . . 273

Arthur Argyle Sagle, On anti-commutative algebras and general Lie triple

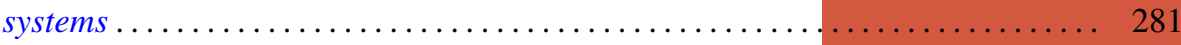

Laurent Siebenmann, A characterization of free projective planes . . . . . . . . . 293

Edward Silverman, Simple areas.................................. 299

James McLean Sloss, Chebyshev approximation to zero .................. 305

Robert S. Strichartz, Isometric isomorphisms of measure algebras . . . . . . . . . 315

Richard Joseph Turyn, Character sums and difference sets . . . . . . . . . . . . 319

L. E. Ward, Concerning Koch's theorem on the existence of arcs . . . . . . . . . . 347

Israel Zuckerman, A new measure of a partial differential field extension ......... 357 